\title{
Research on Grain Refinement and Homogenization during the Mandrel Stretching Process of a Hollow Steel Ingot Used for Heavy Hydro-Generator Shaft
}

\author{
Min LIU ${ }^{\mathrm{a}}$, Qing-Xian MA ${ }^{\mathrm{b}}$, Jian-Bin LUO \\ ${ }^{1}$ Department of Mechanical Engineering, Tsinghua University, Beijing 100084, China \\ aliumin881120@126.com, 'bmaqxdme@mails.tsinghua.edu.cn, 'luojianbin@thpx.org \\ ${ }^{*}$ Corresponding author
}

Keywords: Heavy Forging, Mandrel Stretching, Physical Modeling, Numerical Simulation.

\begin{abstract}
After sequenced rotation with ordinary shaped anvil, there exist coarse grains and mixed grains in the 20Mn5 hollow steel ingot used for hydro-generator shaft. By studying the dynamic recrystallization behavior of 20Mn5 steel under single-pass and multi-pass compression, the criterion for grain refinement in the mandrel stretching process of $20 \mathrm{Mn} 5$ steel is obtained. New shaped anvils and compound shaped anvils for mandrel stretching are proposed, and distribution rules of stress and strain in the forging after single-pass and multi-pass rotatory stretching with different shaped anvils are studied based on physical modeling and numerical simulation methods. Results show strain distribution for new rotation processes with two compound shaped anvils and three new shaped anvils is better than that for sequenced rotation with ordinary V-shaped anvil. The grain refinement effect of big fillet V-shaped anvil-shaped anvils with unequal top and bottom anvil width is best, and the uniformity of convex V-shaped anvil-shaped anvils with unequal top and bottom anvil width is best. It is suggested that three new shaped anvils and two compound shaped anvils be used in the mandrel stretching process of hollow steel ingot used for hydro-generator shaft.
\end{abstract}

\section{Introduction}

Compared with using solid steel ingot for cylinder forging, the advantages of using hollow steel ingot are as follows: it can greatly raise the utilization ratio of steel ingot, avoid punching process and reduce segregation.

A hollow steel ingot used for hydro-generator shaft was forged using mandrel stretching process with upper flat and lower V-shaped anvils. As a lack of knowledge about deformation rules for mandrel stretching, there exist coarse grains and mixed grains in the $20 \mathrm{Mn} 5$ hollow steel ingot after sequenced rotation with ordinary shaped anvil.

Aiming at the void compaction during the stretching process of heavy solid steel ingot, researchers have developed a series of stretching methods such as FM(Free from Mannesmann Effect), JTS(Japan-Tefeno-Shikano) and WHF(Wide Die Heavy Blow Forging)[1-7], and deeply studied recrystallization behaviors of solid steel ingot material[8-12]. However, as the complexity of mandrel stretching process, there have been no reports about the mandrel stretching process of heavy hollow steel ingot.

As the forging ratio of hollow steel ingot is limited, microstructure of hollow steel ingot can't be improved by repeated upsetting and stretching. Hence, new shaped anvils and compound shaped anvils for mandrel stretching are proposed to explore the possibilities of improving strain distribution in the forging and realizing grain refinement and homogenization by improving anvil shape.

\section{Dynamic Recrystallization Behavior and Grain Refinement of 20Mn5 Steel}

As a typical low stacking fault energy steel, dynamic recrystallization can occur in $20 \mathrm{Mn} 5$ steel. Fig. 1 shows the microstructure obtained from 20Mn5 steel compressed to a true strain of 0.7 and quenched in water. Dynamic recrystallization doesn't occur in the rigid zone under the anvils and the corresponding 
grains are coarse, while dynamic recrystallization occurs in the large deformation zone locating in the specimen center and the corresponding grains are fine, uniform and equiaxed.

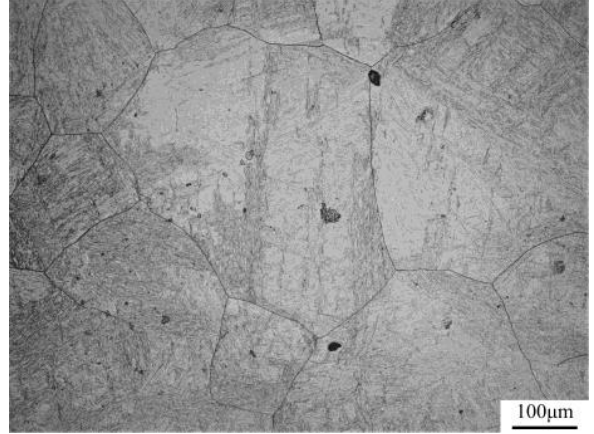

(a) Rigid zone under the anvils

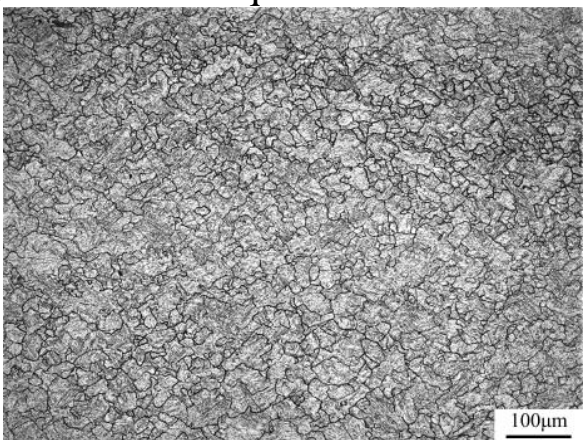

(b) Large deformation zone in the center

Fig. 1 Microstructure of $20 \mathrm{Mn} 5$ steel compressed at strain rate of $0.01 / \mathrm{s}$ and temperature of $900^{\circ} \mathrm{C}$

In actual production of hydro-generator shaft, the strain rate is about 0.01/s. From single-pass compression results the steady state strain and fully dynamically recrystallized grain size at different temperatures and strain rate of $0.01 / \mathrm{s}$ can be obtained, as shown in Tab. 1 .

Tab. 1 Steady state strain and grain size at different temperatures

\begin{tabular}{ccc}
\hline deformation temperature $/{ }^{\circ} \mathrm{C}$ & steady state strain & grain size $/ \mu \mathrm{m}$ \\
\hline 850 & 0.65 & 28.57 \\
\hline 900 & 0.61 & 33.33 \\
\hline 950 & 0.55 & 38.46 \\
\hline 1000 & 0.53 & 47.16 \\
\hline 1050 & 0.49 & 58.82 \\
\hline 1100 & 0.45 & 80.64 \\
\hline 1150 & 0.41 & 90.89 \\
\hline 1200 & 0.37 & 101.48
\end{tabular}

In actual forging process, the forging temperature decreases gradually. The recrystallization behavior of $20 \mathrm{Mn} 5$ steel under double-pass compression were studied by Gleeble1500D. Fig. 2 shows the specimen microstructure after double-pass compression, $\mathrm{T} 1, \varepsilon 1, \mathrm{~T} 2, \varepsilon 2$ are deformation temperatures and strain of the first and second pass respectively. From Fig. 2, it's known that reducing the temperature of the second pass and increasing the strain of the second pass is helpful for obtaining much finer fully dynamically recrystallized grains.

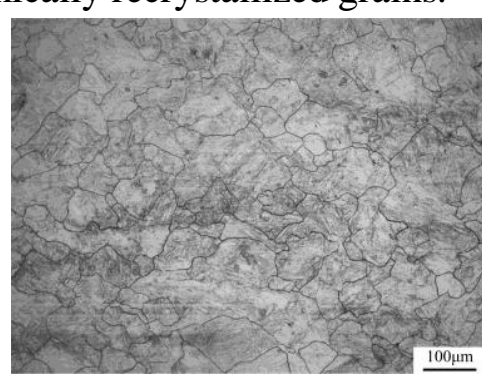

(a) $\mathrm{T} 1=1200^{\circ} \mathrm{C}, \mathrm{T} 2=1150^{\circ} \mathrm{C}$

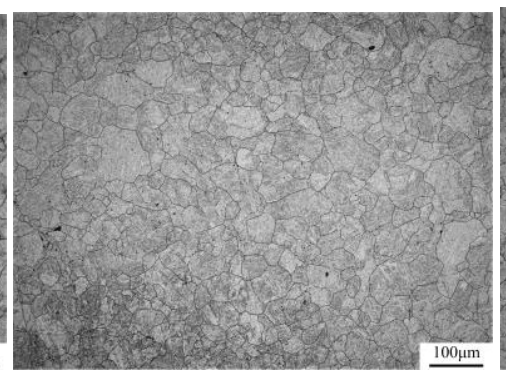

(b) $\mathrm{T} 1=1100^{\circ} \mathrm{C}, \mathrm{T} 2=1050^{\circ} \mathrm{C}$

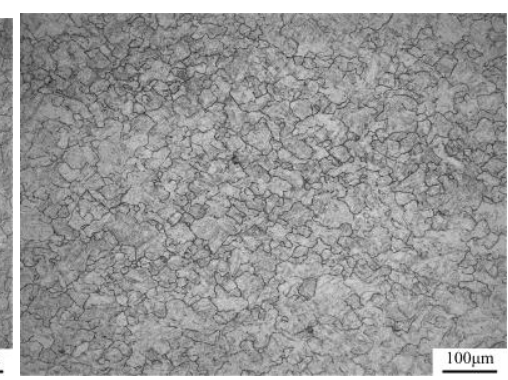

(c) $\mathrm{T} 1=1000^{\circ} \mathrm{C}, \mathrm{T} 2=950^{\circ} \mathrm{C}$

Fig. 2 The microstructure after double-pass compression (strain rate:0.01/s, $\varepsilon 1=0.10$, $\varepsilon 2=0.60$ )

Fig. 3 shows the flow stress curves of $20 \mathrm{Mn} 5$ steel under double-pass compression. When $\varepsilon 1=0.60$ and $\varepsilon 1=0.35$, the flow stress curves of the second pass don't show a steady state. For $\varepsilon 1=0.10$, the flow stress curve of the second pass shows a steady state, and the steady state strain of the second pass is 0.55 which equals the steady state strain of single-pass compression at the same temperature and strain rate. Hence, the steady state strain of the second pass isn't influenced by the first pass. 


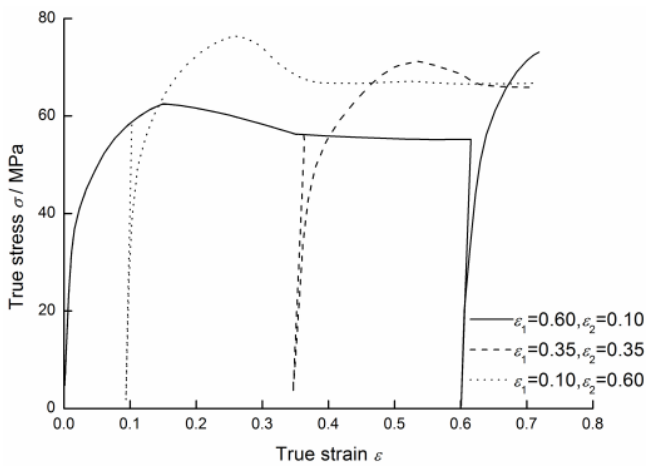

Fig. 3 The flow stress curve of 20Mn5 steel under double-pass compression

At low temperature from $850^{\circ} \mathrm{C}$ to $1050^{\circ} \mathrm{C}$, the deformation resistance of $20 \mathrm{Mn} 5$ steel isn't too high and the fully dynamically recrystallized grain at low temperature is finer(see Table 1). Hence, full dynamic recrystallization at low temperature is the key to realizing grain refinement of $20 \mathrm{Mn} 5$ steel.

Taking full dynamic recrystallization at $850^{\circ} \mathrm{C}$ as a reference, the true strain for the forging process should be more than 0.65 . Meeting the process conditions for full dynamic recrystallization and improving the deformation uniformity is the key to realizing grain refinement and homogenization.

\section{Modeling and Simulation}

\section{Physical Modeling}

Pure lead is the modeling material, and the size of hollow lead specimen is $\Phi 60 \mathrm{~mm} \times \Phi 20 \mathrm{~mm} \times 60 \mathrm{~mm}$. Firstly, the specimen was sliced along the longitudinal section and a grid of $2 \mathrm{~mm} \times 2 \mathrm{~mm}$ was scored on the longitudinal section, then the sliced specimen was bonded by Wood alloy. The mandrel stretching experiment with upper flat and lower $\mathrm{V}$-shaped anvil was conducted on 50t hydraulic testing machine.

\section{Numerical Simulation}

The DEFORM-3D software was used. The hollow steel ingot size is $\Phi 3600 \mathrm{~mm} \times \Phi 1200 \mathrm{~mm} \times 3600 \mathrm{~mm}$; top and bottom anvils, and mandrel are pre-heated to $300^{\circ} \mathrm{C}$; the pressing velocity of top flat anvil is $25 \mathrm{~mm} / \mathrm{s}$, and the reduction ratio is $25 \%$; the friction factor is 0.7 ; heat convection and heat transfer is taken into account; the material for top and bottom anvils, and mandrel is AISI-H13 steel, while the material for hollow steel ingot is $20 \mathrm{Mn} 5$ steel. Fig. 4 shows the coordinate setting for mandrel stretching.

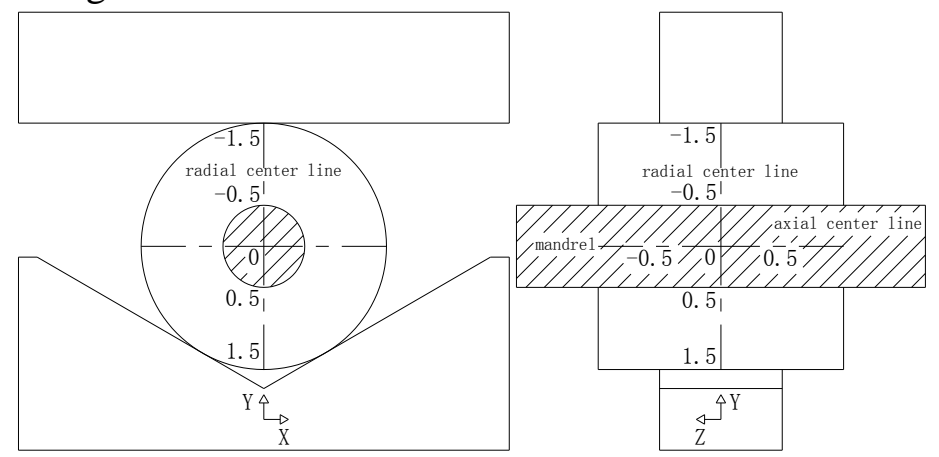

Fig. 4 The coordinate setting for mandrel stretching with upper flat and lower V-shaped anvils

\section{Raise of Three New Shaped Anvils and Anvil Shape Optimization}

\section{Big Fillet V-shaped Anvil}

When using ordinary upper flat and lower V-shaped anvils for mandrel stretching, material tends to flow towards the V-shaped anvil angle gap. After V-shaped anvil angle is changed to be transitional fillet, 
radial material flow is blocked, larger effective strain and mean stress can be obtained. Fig. 5 shows the effective strain distribution for different transitional fillet radii at a reduction ratio of $25 \%$. The lower $\mathrm{V}$-shaped anvil angle is $120^{\circ}$, the anvil width ratio is 0.75 , the original radius of the steel ingot $\mathrm{R}_{0}$ is $1800 \mathrm{~mm}$, the transitional fillet radius is denoted by $\mathrm{R}$ and $\mathrm{R} / \mathrm{R}_{0}$ is transitional fillet radius ratio.
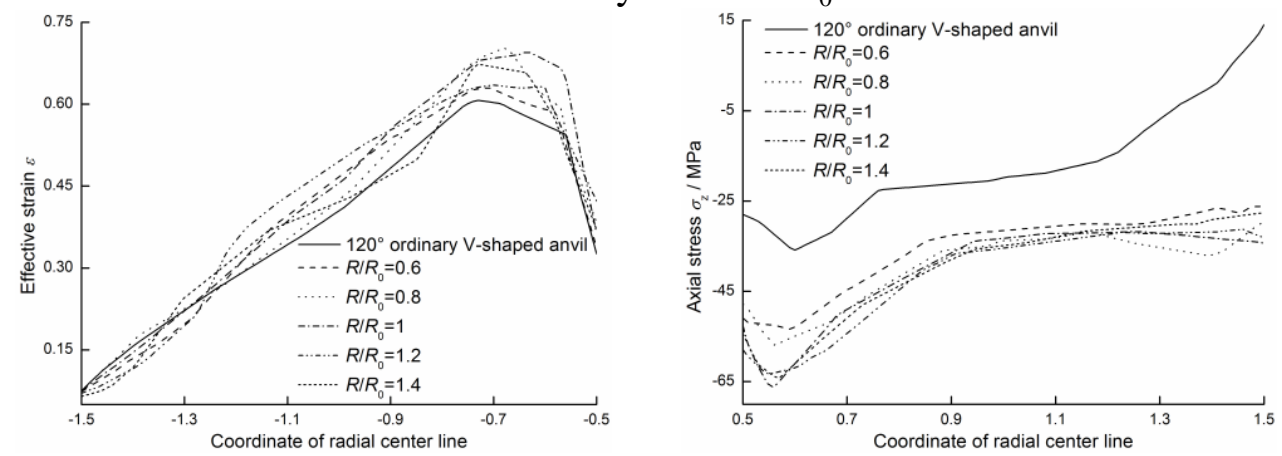

Fig. 5 Effective strain from flat anvil to mandrel Fig. 6 Axial stress from mandrel to V-shaped anvil

In Fig. 5, when R/R0 increases from 0.6 to 1, the effective strain from flat anvil to mandrel increases gradually. When R/R0 is more than 1, the effective strain from flat anvil to mandrel decreases gradually. When R/R0 is 1, the contact area of steel ingot and V-shaped anvil is largest, the largest effective strain can be obtained from flat anvil to mandrel. Moreover, big fillet V-shaped anvil can also eliminate the axial tensile stress at the steel ingot bottom caused by $120^{\circ}$ ordinary $\mathrm{V}$-shaped anvil(see Fig. 6).

Fig. 7 shows the simulation and experimental results for mandrel stretching with $120^{\circ}$ ordinary $\mathrm{V}$-shaped anvil and big fillet $\mathrm{V}$-shaped anvil $(\mathrm{R} / \mathrm{R} 0=0.8)$, the reduction ratio is $25 \%$ and the anvil width ratio is 0.75 . The effective strain field obtained from Fig. 7(c) and Fig. 7(d) is in good agreement with simulation results. Fig. 7(a) and Fig. 7(b) show the strain distribution is greatly improved by big fillet $\mathrm{V}$-shaped anvil. When $\mathrm{R} / \mathrm{R} 0=0.8 \sim 1.2$, larger effective strain and mean stress can be obtained. When $\mathrm{R} / \mathrm{R} 0=1$, the best effect can be achieved.
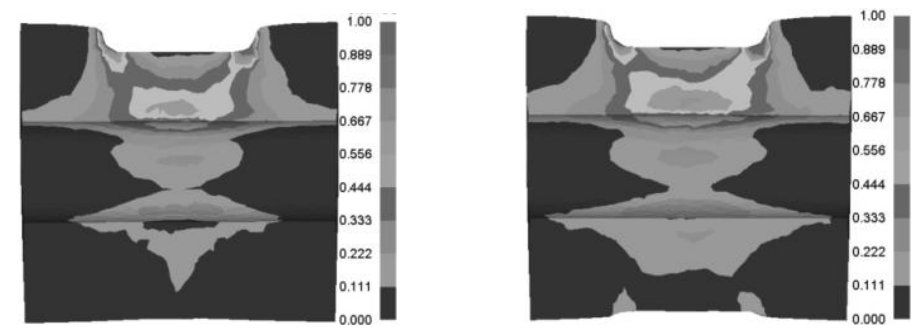

(a) strain for $120^{\circ}$ ordinary V-shaped anvil (b)

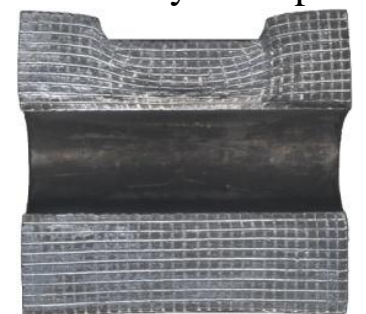

b) strain for $120^{\circ}$ big fillet V-shaped anvil

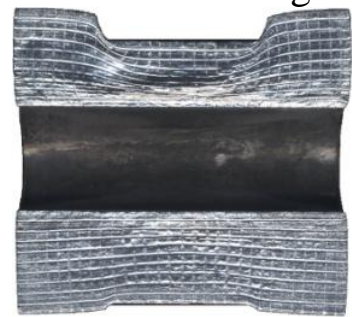

(c) $120^{\circ}$ ordinary $\mathrm{V}$-shaped anvil (d) $120^{\circ}$ big fillet $\mathrm{V}$-shaped anvil

Fig. 7 Comparison of simulation and experimental results for $120^{\circ}$ ordinary V-shaped anvil and big fillet V-shaped anvil

\section{Convex V-Shaped Anvil}

After mandrel stretching with ordinary lower V-shaped anvil, a rigid zone exists on the bottom of the steel ingot (see Fig. 7(a) and Fig. 7(c)), which isn't good for increasing the overall deformation uniformity after rotatory stretching. To increase the effective strain for the steel ingot center and surface, and improve the deformation uniformity for one-time stretching, the ordinary $\mathrm{V}$-shaped anvil angle is changed to be a convex circular arc surface, and a new convex V-shaped anvil is proposed. The main dimensions of 
convex V-shaped anvil are shown in Fig. 8, Rc is the convex fillet radius, transitional fillet radius $\mathrm{r}=0.1 \mathrm{~W}$ and $\mathrm{W}$ is anvil width.

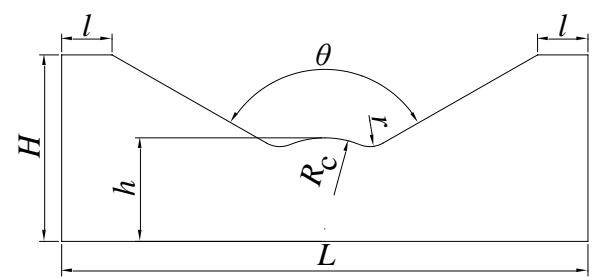

Fig. 8 The main dimensions of convex V-shaped anvil

Fig. 9 shows the effective strain distribution for mandrel stretching with different convex fillet radii at a reduction ratio of $25 \%$. The lower $\mathrm{V}$-shaped anvil angle is $120^{\circ}$, the anvil width ratio is $0.75, \mathrm{Rc} / \mathrm{R} 0$ is the convex fillet radius ratio of the lower $\mathrm{V}$-shaped anvil. Fig. 9 shows when $0.1 \leq \mathrm{Rc} / \mathrm{R} 0 \leq 0.5$, the peak effective strain from flat anvil to mandrel is more than that of $120^{\circ}$ ordinary V-shaped anvil.

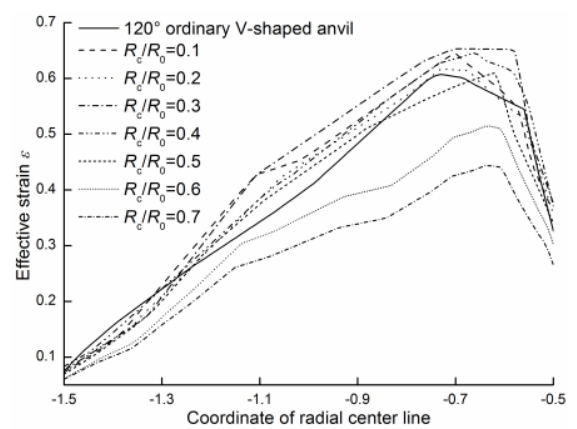

Fig. 9 Effective strain from flat anvil to mandrel

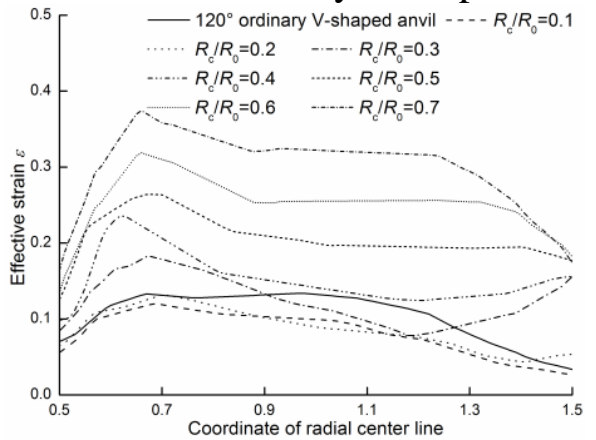

Fig. 10 Effective strain from mandrel to $\mathrm{V}$ anvil

As shown in Fig. 10, when $\mathrm{Rc} / \mathrm{R} 0 \geq 0.3$, the effective strain from mandrel to $\mathrm{V}$-shaped anvil is significantly larger than that of $120^{\circ}$ ordinary V-shaped anvil. The convex V-shaped anvil greatly improves the deformation uniformity of the forging. Rc/R0 should range from 0.3 to 0.5 to ensure convex $\mathrm{V}$-shaped anvil has good compaction effect and deformation uniformity. When $\mathrm{Rc} / \mathrm{R} 0=0.4$, the stretching effect is best.

\section{Shaped Anvils with Unequal Top and Bottom Anvil Width}

Larger anvil width ratio is good for obtaining larger effective strain, higher mean stress and higher stretching efficiency. But too large anvil width ratio can cause large tensile stress and high press load. Inspired by FM method, a kind of shaped anvils with unequal top and bottom anvil width is proposed. The anvil width ratio of the upper flat anvil is 0.75 . Fig. 11 shows the effective strain and axial displacement of end surface from flat anvil to mandrel for different anvil width ratios of lower V-shaped anvils at a reduction ratio of $25 \%$. The anvil width ratio of upper flat anvil is 0.75 and the lower $\mathrm{V}$-shaped anvil angle is $120^{\circ}$.

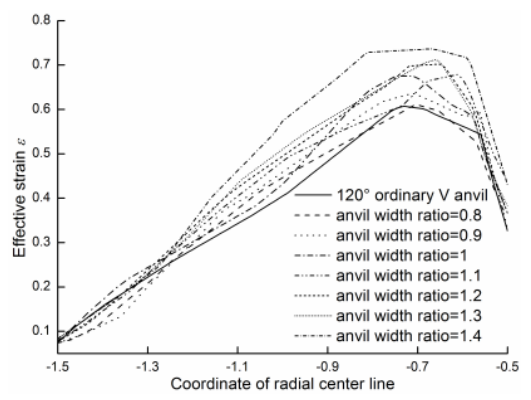

(a) effective strain

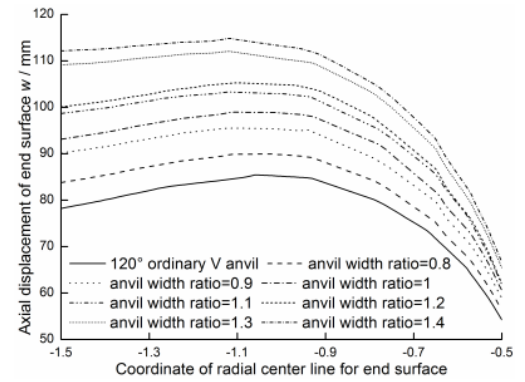

(b) axial displacement of end surface

Fig. 11 From flat anvil to mandrel

Fig. 11(a) shows the larger the anvil width ratio of the lower V-shaped anvil, the larger the peak effective strain. And large anvil width ratio of lower V-shaped anvil can increase stretching efficiency(see 
Fig. 11(b)). But when anvil width ratio of lower V-shaped anvil is more than 1.1, large tangential tensile stress forms on the bottom of the steel ingot. Hence, the anvil width ratio of the lower V-shaped anvil should be in the range of $0.9 \sim 1.1$. When anvil width ratio of the lower $\mathrm{V}$-shaped anvil is 1.1, the stretching effect is best.

\section{Raise of Compound Shaped Anvil and Comparison of Stretching Effect}

To make full use of the advantages of three new shaped anvils, two compound shaped anvils(big fillet $\mathrm{V}$-shaped anvil-shaped anvils with unequal top and bottom anvil width(R/R0=1.0, anvil width ratio of lower V-shaped anvil is 1.1), convex V-shaped anvil-shaped anvils with unequal top and bottom anvil width(Rc/R0=0.4, anvil width ratio of the lower $\mathrm{V}$-shaped anvil is 1.1$)$ ) are proposed. Fig. 12 shows the peak effective strain for compound shaped anvils and new shaped anvils is larger than that for $120^{\circ}$ ordinary V-shaped anvil. In Table 2, big fillet V-shaped anvil-shaped anvils with unequal top and bottom anvil width, convex $\mathrm{V}$-shaped anvil-shaped anvils with unequal top and bottom anvil width are denoted by 1.0-1.1 and 0.4-1.1, respectively. Table 2 shows deformation between mandrel and V-shaped anvil is small, the deformation uniformity for convex V-shaped anvil-shaped anvils with unequal top and bottom anvil width is best. In terms of compaction effect and deformation uniformity under one-time stretching, the two compound shaped anvils are better than three new shaped anvils, three new shaped anvils are better than $120^{\circ}$ ordinary V-shaped anvil.

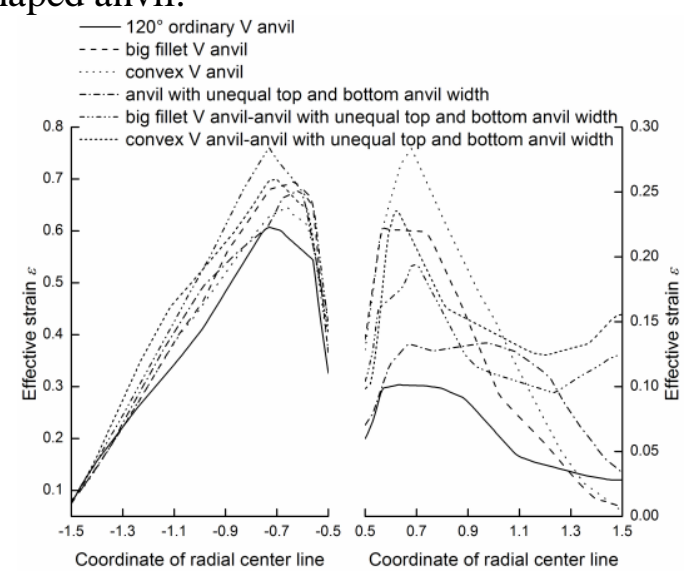

Fig. 12 Strain distribution along radial center line for different shaped anvils at a reduction ratio of $25 \%$

Tab. 2 Average value and standard deviation of strain at a reduction ratio of $25 \%$

\begin{tabular}{cccc}
\hline shaped anvils & $\begin{array}{c}\text { average strain between } \\
\text { flat anvil and mandrel }\end{array}$ & $\begin{array}{c}\text { average strain between } \\
\text { mandrel and V anvil }\end{array}$ & standard deviation \\
\hline $120^{\circ}$ ordinary V anvil & 0.385 & 0.065 & 0.226 \\
\hline big fillet V anvil & 0.432 & 0.115 & 0.206 \\
\hline convex V anvil & 0.411 & 0.141 & 0.203 \\
\hline $\begin{array}{c}\text { anvil with unequal top } \\
\text { and bottom anvil width }\end{array}$ & 0.425 & 0.106 & 0.218 \\
\hline $1.0-1.1$ & 0.469 & & 0.197 \\
\hline $0.4-1.1$ & 0.461 & 0.129 & 0.194 \\
\hline
\end{tabular}

\section{Rotatory Stretching Results for Different Shaped Anvils and Analysis}

Fig. 13 shows strain distribution on the circular section of the forging after rotatory stretching at a reduction ratio of $25 \%$. Obviously, the deformation uniformity of three new shaped anvils and two compound shaped anvils is better than that of ordinary V-shaped anvil, and three new shaped anvils and two compound shaped anvils eliminate the small deformation zone, which is good for full dynamic recrystallization to occur in $20 \mathrm{Mn} 5$ steel. 

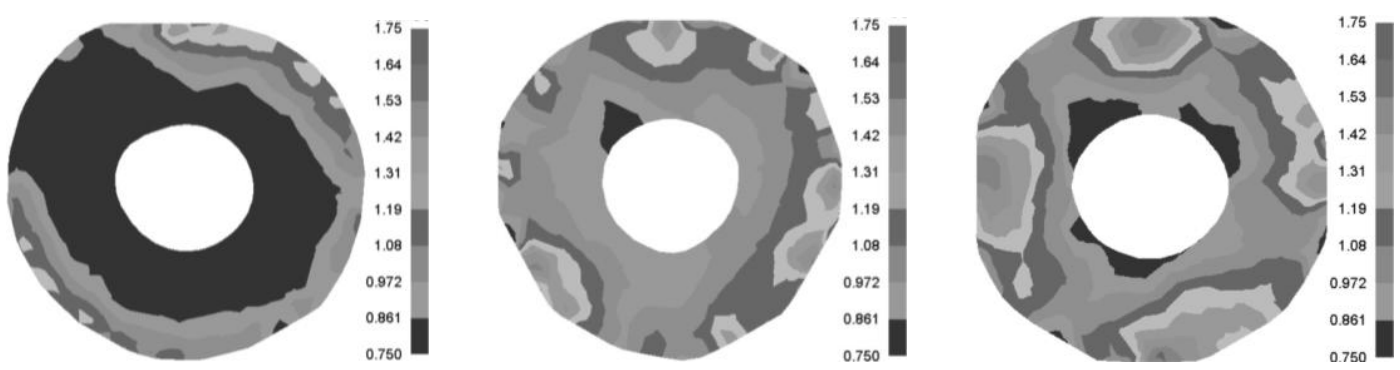

(a) ordinary $\mathrm{V}$ anvil (b) convex $\mathrm{V}$ anvil (c) anvil with unequal top and bottom anvil width
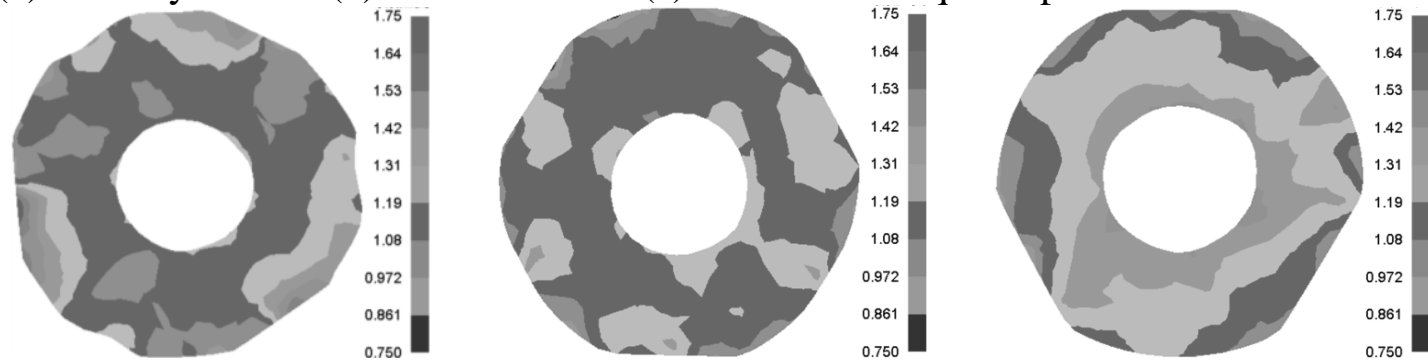

(d) big fillet $\mathrm{V}$ anvil (e) convex $\mathrm{V}$ anvil-anvil with unequal top and bottom anvil width (f) big fillet $\mathrm{V}$ anvil-anvil with unequal top and bottom anvil width

Fig. 13 Strain distribution on the circular section of the forging after rotatory stretching

As known from Fig. 14(a), the strain at the place 0.5 time of the steel ingot thickness away from outer wall for ordinary $\mathrm{V}$-shaped anvil is smallest, and along the circular direction the strain of about 1/7 part is less than 0.65 required. The circular deformation uniformity and compaction effect for new shaped anvils and compound shaped anvils is obviously better than that for ordinary V-shaped anvil. The strain distribution rule at other place is like this place. Fig. 14(b) shows after sequenced rotation with ordinary V-shaped anvil, the strain from inner wall to the place 0.3 time of the steel ingot thickness away from the inner wall is less than 0.65 required, and the radial deformation is uneven. The radial deformation uniformity and compaction effect for new shaped anvils and compound shaped anvils is better.
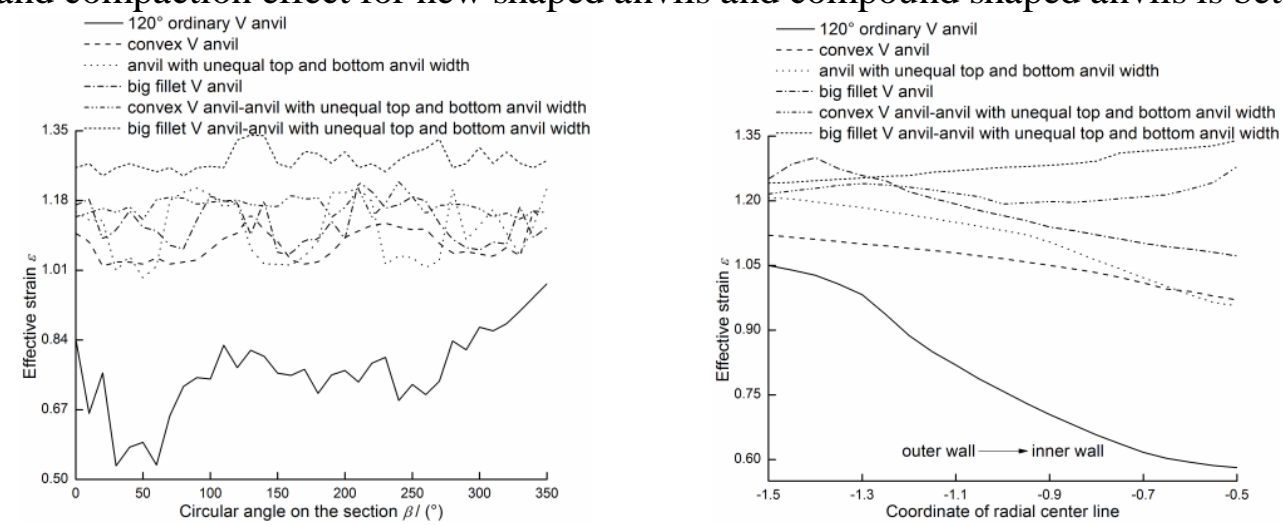

(a) circular strain at the place 0.5 time of the ingot thickness away from outer wall (b) radial strain

Fig. 14 The circular and radial strain distribution on the forging section after rotatory stretching

" 0.5 circular" stands for the circular direction 0.5 time of the steel ingot thickness away from the outer wall in Fig. 15. Fig. 15 shows the average strain for big fillet V-shaped anvil-shaped anvils with unequal top and bottom anvil width is the largest in different directions, and Fig. 13(f) shows the strain on the circular section is more than 0.65 . The average strain for ordinary V-shaped anvil is smallest, and full dynamic recrystallization can't occur in some area. So the grain refinement effect for big fillet V-shaped anvil-shaped anvils with unequal top and bottom anvil width is best; the standard deviation of strain for convex V-shaped anvil-shaped anvils with unequal top and bottom anvil width is the least in different directions, the standard deviation of strain for ordinary $\mathrm{V}$-shaped anvil is the largest, so the uniformity for convex V-shaped anvil-shaped anvils with unequal top and bottom anvil width is best. 


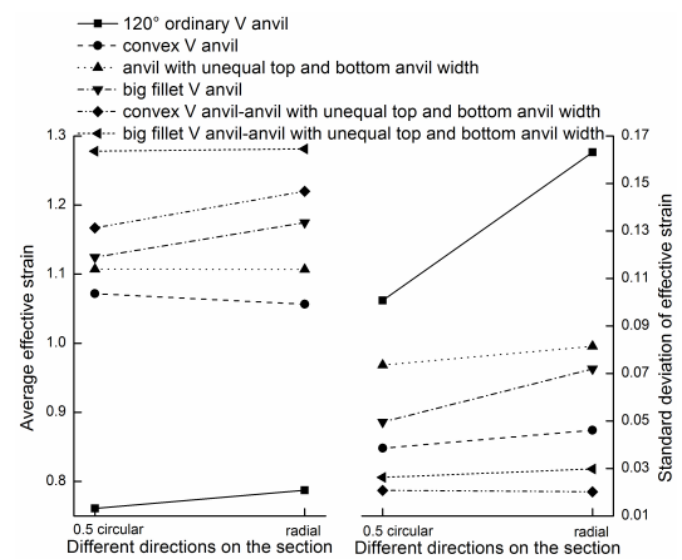

Fig. 15 Average value and standard deviation of circular and radial strain on the section after rotatory stretching

\section{Conclusions}

(1) The grain can be refined by full dynamic recrystallization on condition that true strain of the forging process is more than 0.65 .

(2) Three new shaped anvils are proposed. All of the three new shaped anvils have improved the strain distribution in the hollow steel ingot. The fillet radius ratio of big fillet $\mathrm{V}$-shaped anvil should be in the range of $0.8 \sim 1.2$; the fillet radius ratio of the convex $\mathrm{V}$-shaped anvil should range from 0.3 to 0.5 ; When the anvil width ratio of upper flat anvil is 0.75 , the anvil width ratio of lower $\mathrm{V}$-shaped anvil should be in the range of $0.9 \sim 1.1$.

(3) Two compound shaped anvils(big fillet V-shaped anvil-shaped anvils with unequal top and bottom anvil width, convex $\mathrm{V}$-shaped anvil-shaped anvils with unequal top and bottom anvil width) are proposed. The compaction effect and deformation uniformity for two compound shaped anvils is better than three new shaped anvils.

(4) Rotatory stretching results show strain distribution for new rotation process of two compound shaped anvils and three new shaped anvils is better than that for sequenced rotation of ordinary V-shaped anvil. The grain refinement effect for big fillet $\mathrm{V}$-shaped anvil-shaped anvils with unequal top and bottom anvil width is best. The uniformity for convex V-shaped anvil-shaped anvils with unequal top and bottom anvil width is best.

\section{References}

[1] X.X. Zhang, Z.S. Cui and W. Chen, A criterion for void closure in large ingots during hot forging, J. Mater. Process. Technol. 209 (2009) 1950-1959.

[2] H. Kakimoto, T. Arikawa and Y. Takahashi, Development of forging process design to close internal voids, J. Mater. Process. Technol. 210 (2010) 415-422.

[3] Y.D. Kim, J.R. Cho and W.B. Bae, Efficient forging process to improve the closing effect of the inner void on an ultra-large ingot, J. Mater. Process. Technol. 211 (2011) 1005-1013.

[4] Y.S. Lee, S.U. Lee and C.J. Van Tyne, Internal void closure during the forging of large cast ingots using a simulation approach, J. Mater. Process. Technol. 211 (2011) 1136-1145.

[5] M.S. Chen and Y.C. Lin, Numerical simulation and experimental verification of void evolution inside large forgings during hot working, Int. J. Plast. 49 (2013) 53-70.

[6] M.S. Chen, Y.C. Lin and K.H. Chen, Evolution of elliptic-cylindrical and circular-cylindrical voids inside power-law viscous solids, Int. J. Plast. 53 (2014) 206-227.

[7] W. Chen and Z.S. Cui, New method for finite element simulation of stretch forming, J. Mech. Eng. 46 (2010) 40-46. 
[8] F. Chen, Z.S. Cui and S.J. Chen, Recrystallization of 30Cr2Ni4MoV ultra-super-critical rotor steel during hot deformation. Part I: Dynamic recrystallization, Mater. Sci. Eng. A. 528 (2011) 5073-5080.

[9] F. Chen, Z.S. Cui and D.S. Sui, Recrystallization of 30Cr2Ni4MoV ultra-super-critical rotor steel during hot deformation. Part III: Metadynamic recrystallization, Mater. Sci. Eng. A. 540 (2012) 46-54.

[10]F. Chen, K. Qi and Z.S. Cui, Modeling the dynamic recrystallization in austenitic stainless steel using cellular automaton method, Comput. Mater. Sci. 83 (2014) 331-340.

[11]D.Q. Dong, F. Chen and Z.S. Cui, A physically-based constitutive model for SA508-III steel: Modeling and experimental verification, Mater. Sci. Eng. A. 634 (2015) 103-115.

[12] M.S. Chen, Y.C. Lin and X.S. Ma, The kinetics of dynamic recrystallization of 42CrMo steel, Mater. Sci. Eng. A. 556 (2012) 260-266. 